\title{
Integrals of motion and quantum operators for hydrogenic atoms in external fields
}

\author{
Marcus W. Beims ${ }^{1}$ and Jason A. C. Gallas ${ }^{2,3}$ \\ ${ }^{1}$ Departamento de Física, Universidade Federal do Paraná, 81531-990 Curitiba, Brazil \\ ${ }^{2}$ Departamento de Física, Faculdade de Ciências, Universidade de Lisboa, 1749-016 Lisboa, Portugal \\ ${ }^{3}$ Instituto de Física, Universidade Federal do Rio Grande do Sul, 91501-970 Porto Alegre, Brazil
}

(Received 06 April 2000; published 19 September 2000)

\begin{abstract}
We report five cases of integrability for a hydrogenic atom under three static external fields: a magnetic field, an electric field, and a van der Waals interaction. Exact integrals of motion and corresponding quantum operators are obtained explicitly for each case. Integrals of motion (quantum operators) can be expressed as components of a suitably generalized Runge-Lenz vector (operator). Quadratic quantum operators are found to have the amazing property of requiring a nonclassical extra term proportional to $\hbar^{2}$. The structuring of the classical phase space is investigated numerically via Poincare surfaces of section and corroborates the analytical results.

PACS number(s): 32.60.+i, 03.65.-w, 31.15.-p
\end{abstract}

\section{INTRODUCTION}

The investigation of the behavior of a hydrogen atom in a static magnetic field has been a source of many exciting results in atomic physics during the last two decades [1,2]. This is so because the quadratic Zeeman effect leads to a complicated nonseparable problem which, for suitable parameters, displays deterministic chaotic behavior, displays quasi-Landau resonances, is not easily quantizable, etc. A hydrogen atom in external magnetic fields provides a paradigmatic Hamiltonian system because of the decisive advantage of allowing its dynamics to be investigated experimentally $[1-8]$. Atomic physics in the presence of external fields offers a few additional situations where recent developments of laser spectroscopy can be used advantageously to probe experimentally the effects of chaotic dynamics, e.g., in cold atoms $[9,10]$, in hydrogen atoms in a microwave field $[1,11]$, in a resonant tunneling diode $[12,13]$, in a helium atom $[14,15]$, in the spreading and dispersion of initially localized wave packets [16], in quantum dots, and in many other situations in external fields [2].

Most nonlinear problems involving atoms and magnetic fields are very hard to investigate analytically, and general solutions are difficult to obtain. Exceptionally, however, for suitable combinations of parameters, one may be able to integrate the equations of motion, i.e., to derive exact solutions. The explicit location of these suitable parameters, the integrable cases, can be rather tricky. Knowledge of integrable cases is important because, by perturbing them, one obtains insight about the behavior of nearby living nonintegrable states. For example, sets of known solutions are very often useful bases for perturbative and asymptotic expansions and analytic continuation [17]. Although it is sometimes useful to consider "local dynamics" and to solve the equations of motion for specific trajectories, it is important to keep in mind that the quadratic Zeeman effect always remains a nonseparable contribution, implying that onedimensional solutions are just approximations.

The purpose of the present paper is to report the results of a systematic investigation of the parameter space of a rather general nonrelativistic hydrogenic Hamiltonian, looking for suitable combinations of control parameters which render such a Hamiltonian integrable. We start by searching for good candidates for integrability by performing a standard Painlevé analysis [18]. Then, for each candidate, we either rule it out as being nonintegrable or, if integrable, explicitly construct classical integrals of motion as well as corresponding quantum operators, without approximations. Altogether, we find five new integrals of motions. An interesting finding is that the quadratic quantum operators associated with the integrable cases require an additional term proportional to $\hbar^{2}$ which, obviously, is absent in the classical limit. Analogous $\hbar^{2}$-dependent terms were found in a different context, among classes of duality transformations interconnecting Hamiltonian systems [19]. To find such $\hbar^{2}$ corrections is not totally trivial, since different quantum systems may share a common classical limit [19], a quite interesting fact.

The nonrelativistic hydrogenic Hamiltonian studied here is (in units such that $\mu=\hbar=e=a_{0}=1$ )

$$
\hat{H}=\frac{1}{2} \hat{\mathbf{p}}^{2}-\frac{Z}{\hat{r}}+\hat{V}_{\text {ext }},
$$

where $\hat{r}^{2}=\hat{q}_{1}^{2}+\hat{q}_{2}^{2}+\hat{q}_{3}^{2}, \hat{\mathbf{p}}^{2}=\hat{p}_{1}^{2}+\hat{p}_{2}^{2}+\hat{p}_{3}^{2}$, and the term proportional to $Z$ corresponds to a generic hydrogenic core which for $Z=1$ reduces to the hydrogen atom, as usual. The external potential $\hat{V}_{\text {ext }}$ considered is

$$
\hat{V}_{e x t}=\gamma\left(\hat{q}_{1}^{2}+\hat{q}_{2}^{2}+\beta \hat{q}_{3}^{2}\right)+F \hat{q}_{3},
$$

where $\gamma, F$, and $\beta$ are independent control parameters representing the magnitude of the applied external fields: $\gamma$, measured in units $B_{0}=4.72 \times 10^{5} \mathrm{~T}$, controls the quadratic Zeeman effect; $F$, in units of $F_{0}=5.14 \times 10^{11} \mathrm{~V} / \mathrm{cm}$, controls the Stark effect; and the adimensional number $\beta$ controls the anharmonicity associated to the van der Waals interaction [20-22]. This combined potential is very interesting because of its similarity to the fields seen by an ion confined in a Paul trap [23].

The paper is organized as follows. Section II briefly reviews the derivation of the regularized equations of motion 
for electronic motions around a hydrogenic core. Section III presents a Painlevé analysis which reveals the particular sets of parameters that are possible candidates for integrability. In Sec. IV we discriminate those candidates which really lead to integrability and, for each of them, construct corresponding integrals of motion. We also investigate numerically via Poincare surfaces of section the structure of the phase space for the integrable cases, comparing them with nearby living nonintegrable solutions of the regularized equations of motion. In Sec. V we derive the quantum operators associated with the classical integrals. Finally, Sec. VI contains our conclusions.

\section{EQUATIONS OF MOTION}

A popular and useful simplification in the study of a hydrogen atom subjected to external fields is to consider a twodimensional "regularized" version of the real threedimensional system [24]. The main reasons for this approach are that (i) a two-dimensional (2D) problem is computationally simpler to deal with than a 3D one; and (ii) by "removing" the Coulomb singularity from the original dynamical system, the regularization procedure makes the numerical work easier. A further convenient aspect is that the regularized 2D Hamiltonian involves two coupled anharmonic oscillators, a system for which one possesses efficient tools for extracting spectral information. These nice characteristics have made the regularization procedure the standard way of obtaining the Hamiltonian on which virtually all theoretical investigations of the quadratic Zeeman effect have been done to date. Note that $\hat{L}_{q_{3}}$, the component of the angular momentum along the magnetic field direction, always remains a good quantum number when magnetic and electric fields are parallel.

As a preliminary to the Painlevé analysis, we derive now the classical regularized 2D Hamiltonian corresponding to the original 3D problem (1). First, we transform each operator of Eq. (1) in the corresponding classical one, i.e., $\hat{q}_{i}$ $=q_{i}$ and $\hat{p}_{q_{i}}=p_{q_{i}}$, for $i=1,2$, and 3 . The regularized Hamiltonian is then obtained by transforming Eq. (1) to semiparabolic coordinates, namely,

$$
\begin{aligned}
& q_{1}=u v \cos (\phi), \\
& q_{2}=u v \sin (\phi), \\
& q_{3}=\frac{1}{2}\left(u^{2}-v^{2}\right),
\end{aligned}
$$

and the corresponding momenta

$$
\begin{aligned}
& p_{q_{1}}=\frac{p_{v} u \cos (\phi)}{u^{2}+v^{2}}+\frac{p_{u} v \cos (\phi)}{u^{2}+v^{2}}-\frac{p_{\phi} \sin (\phi)}{u v}, \\
& p_{q_{2}}=\frac{p_{\phi} \cos (\phi)}{u v}+\frac{p_{v} u \sin (\phi)}{u^{2}+v^{2}}+\frac{p_{u} v \sin (\phi)}{u^{2}+v^{2}}
\end{aligned}
$$

$$
p_{q_{3}}=\frac{p_{u} u-p_{v} v}{u^{2}+v^{2}}
$$

After such transformation, the regularized Hamiltonian reads

$$
\mathcal{H}(u, v)=2\left(u^{2}+v^{2}\right)[H(u, v)-E]
$$

or, more explicitly,

$$
\begin{aligned}
\mathcal{H}(u, v)=2 Z= & \frac{1}{2}\left(p_{u}^{2}+p_{v}^{2}\right)+\frac{\epsilon}{2}\left(u^{2}+v^{2}\right)+\frac{L_{q_{3}}^{2}}{2}\left(\frac{1}{u^{2}}+\frac{1}{v^{2}}\right) \\
& +A\left(u^{6}+v^{6}\right)+B\left(u^{4} v^{2}+u^{2} v^{4}\right) \\
& +C\left(v^{4}-u^{4}\right)
\end{aligned}
$$

where $\epsilon=-2 E, A=\gamma \beta / 4, B=\gamma(4-\beta) / 4$, and $C=F / 2$. In this equation, $L_{q_{3}}$ is a conserved quantity and we put $L_{q_{3}}$ $=m$, with $m$ a constant.

The equations of motion associated with $\mathcal{H}(u, v)$ are, therefore,

$$
\begin{aligned}
& \ddot{u}=-\epsilon u+\frac{m^{2}}{u^{3}}-6 A u^{5}-B\left(4 u^{3} v^{2}+2 u v^{4}\right)+4 C u^{3}, \\
& \ddot{v}=-\epsilon v+\frac{m^{2}}{v^{3}}-6 A v^{5}-B\left(2 u^{4} v+4 u^{2} v^{3}\right)-4 C v^{3}
\end{aligned}
$$

The only formal difference between the Hamiltonian of an ion in a Paul trap is that while the total energy of the Hamiltonian (6) is $2 Z$, that of a trapped ion is -2 (for details, see Ref. [22]).

\section{PAINLEVÉ ANALYSIS}

The Painlevé analysis is a powerful technique to uncover integrability candidates for differential equations in general [18]. In this section we present the results of the Painlevé analysis for the equations of motion (7) and (8). A similar analysis for the same problem was previously done in Ref. [21] which, however, considered the case without electric field $(C=F / 2=0)$ and $m=0$. Briefly, the analysis consists of three steps: (i) to find the dominant term of each equation of motion; (ii) to find their resonances; and (iii) to determine the number of arbitrary constants in series expansions of the solutions of the equation of motion. A given equation satisfies the Painlevé conjecture and, therefore, generates a set of candidate parameters for which integrability might exist when, apart from -1 , all additional resonances are positive integers and the aforementioned series expansion contains a suitable number of arbitrary constants, four in the case of Eqs. (7) and (8).

Abbreviating $\tau \equiv t-t_{0}$, and substituting

$$
u=a_{10} \tau^{k_{1}} \text { and } v=a_{20} \tau^{k_{2}}
$$

into Eqs. (7) and (8), yields 


$$
\begin{aligned}
k_{1}\left(k_{1}-1\right) a_{10} \tau^{k_{1}-2} & \\
= & -\epsilon a_{10} \tau^{k_{1}}+\frac{m^{2}}{a_{10}^{3}} \tau^{-3 k_{1}}-B\left(4 a_{10}^{3} a_{20}^{2} \tau^{3 k_{1}+2 k_{2}}\right. \\
& +2 a_{10} a_{20}^{4} \tau^{\left.k_{1}+4 k_{2}\right)} \\
& -6 A a_{10}^{5} \tau^{5 k_{1}}+4 C a_{10}^{3} \tau^{3 k_{1}}, \\
k_{2}\left(k_{2}-1\right) a_{20} \tau^{k_{2}-2} & \\
= & \epsilon a_{20} \tau^{k_{2}}+\frac{m^{2}}{a_{20}^{3}} \tau^{-3 k_{2}-B}\left(2 a_{10}^{4} a_{20} \tau^{4 k_{1}+k_{2}}\right. \\
+ & \left.4 a_{10}^{2} a_{20}^{3} \tau^{2 k_{1}+3 k_{2}}\right)-6 A a_{20}^{5} \tau^{5 k_{2}-4 C a_{20}^{3} \tau^{3 k_{2}},}
\end{aligned}
$$

corresponding to Ansätze for solutions near $t=t_{0}$.

For particular choices of $k_{1}$ and $k_{2}$, some terms of the equations balance when $t \rightarrow t_{0}$, while others can be ignored because of their much smaller magnitudes. The terms which balance are called 'dominant' terms of the equation.

The detailed analysis of Eqs. (10) and (11) involves finding, for each doublet $\left(k_{1}, k_{2}\right)$ that satisfies the equations, the dominant terms in the limit $\tau \rightarrow 0$ and, from them, obtaining conditions that constrain the parameters of the model. Such an analysis is long and tedious. We present only the final results.

The only case which satisfies the three steps of the Painlevé analysis is $k_{1}=k_{2}=-1 / 2$, the dominant terms in Eqs. (10) and (11) then being those proportional to $\tau^{-5 / 2}$, which yield

$$
\begin{aligned}
& \frac{3}{4}+6 A a_{10}^{4}+4 B a_{10}^{2} a_{20}^{2}+2 B a_{20}^{4}=0 \\
& \frac{3}{4}+6 A a_{20}^{4}+4 B a_{10}^{2} a_{20}^{2}+2 B a_{10}^{4}=0
\end{aligned}
$$

Subtracting Eq. (12) from Eq. (13) we obtain

$$
2(3 A-B)\left(a_{10}^{4}-a_{20}^{4}\right)=0 .
$$

Essentially, this equation has two solutions (i) $B=3 A$ and (ii) $a_{10}^{4}=a_{20}^{4}$.

For $B=3 A$, we obtain

$$
a_{20}^{2}=-a_{10}^{2} \pm \sqrt{-1 /(8 A)},
$$

the corresponding resonances being $-1,3,0$, and 2 . For $a_{20}^{4}$ $=a_{10}^{4}$ we might have either $a_{20}^{2}=+a_{10}^{2}$ or $a_{20}^{2}=-a_{10}^{2}$, when Eqs. (12) and (13) lead to

$$
a_{10}^{4}=-\frac{1}{8(A+B)}
$$

and

$$
a_{10}^{4}=-\frac{3}{8(3 A-B)},
$$

respectively. The corresponding resonances are

$$
-1,3,1+2 \sqrt{\frac{A}{A+B}} \text { and } 1-2 \sqrt{\frac{A}{A+B}}
$$

and

$$
-1,-1,3 \text {, and } 3 \text {. }
$$

The square root in Eq. (18) considerably restricts the possible choices of $A$ and $B$ if the resonances are to be positive integers as required by the Painlevé analysis. Let us discuss the resonances arising from these square roots when $m=0$. As seen from Eqs. (7) and (8), for $B=0$ the system decouples being thus obviously integrable.

For $A=0$, both square roots give 1 as the resonance, corresponding physically to either (a) $\gamma=0$, thereby reducing the problem to a pure hydrogen atom in an electric field, or (b) $\beta=0$, and arbitrary $\gamma$, reducing the problem to the quadratic Zeeman effect with a parallel electric field added. This second case, however, does not survive the third step of the Painlevé analysis.

For $B=3 A$ the resonances are 0 and 2 and survive the third step. For $B=8 A$ the resonances are $1 / 3$ and $5 / 3$, and are not admissible because they are not positive integers. Then, instead of the Painlevé property, one could still have the so-called weak Painlevé property [25], with series expansions around $1 / 3$. This possibility is ruled out because we already have $k_{1}=1 / 2$.

For $B=15 A$ the resonances are $1 / 2$ and $3 / 2$; the system satisfies the weak Painlevé property, and can be expanded in powers of $1 / 2$. From the third step we find that if $B=15 A$, then we must have the additional condition $C=0$.

All subsequent possibilities, namely, $B=24 A, B=35 A$, $\ldots, B=(n-1) A$ lead to resonances $(3 / 5,7 / 5),(2 / 3,4 / 3)$, $\ldots,(1-2 / \sqrt{n}, 1+2 / \sqrt{n})$, respectively. From this we do not expect further additional cases satisfying the Painlevé analysis.

Summarizing, we find the following candidates for integrability: (a) $B=3 A, C$ arbit., implying $A=\gamma / 4$ and $\beta=1$; (b) $B=0, C$ arbit., implying $A=\gamma$ and $\beta=4$; and (c) $B$ $=15 A, C=0$, implying $A=\gamma / 16$ and $\beta=\frac{1}{4}$. For $C=0$, the results above coincide with those found earlier in Ref. [21].

\section{CLASSICAL INTEGRALS OF MOTION}

We now derive the classical integrals of motion for Hamiltonian (1) following a very useful procedure used by Redmond [26]. We start observing that the equations of motion may be written as

$$
\frac{d \mathbf{p}}{d t}=-\frac{Z \hat{\mathbf{r}}}{r^{2}}-\nabla V_{e x t},
$$

where $\hat{r}$ denotes a unitary vector, and

$$
\boldsymbol{\nabla} V_{e x t}=\frac{\partial V_{e x t}}{\partial x} \hat{\mathbf{i}}+\frac{\partial V_{e x t}}{\partial y} \hat{\mathbf{j}}+\frac{\partial V_{e x t}}{\partial z} \hat{\mathbf{k}}
$$


The angular momentum is $\mathbf{L}=\mathbf{r} \times \mathbf{p}$, and obeys

$$
\frac{d}{d t} \mathbf{L}=\mathbf{r} \times \frac{d \mathbf{p}}{d t}=\nabla V_{e x t} \times \mathbf{r}=\left[2 \gamma(\beta-1) q_{3}+F\right][\hat{k} \times \mathbf{r}] .
$$

The square brackets in this last equation mean that (i) $\mathbf{L}$ is an integral of motion when, simultaneously, $\beta=1$ and $F=0$, or

(ii) $L_{q_{3}}$, the $q_{3}$ component (along $\hat{k}$ ) of $\mathbf{L}$, will be always an integral of motion.

Observe that the original Hamiltonian [Eq. (1)] has three degrees of freedom, and that we already have two integrals of motion, namely, the total energy and $L_{q_{3}}$. Therefore, we just need to find one additional integral of motion to show that system (1) is integrable for a given set of parameters $\gamma$, $\beta$, and $F$. We refer to Ref. [17] for details on the conditions for integrability.

The key observation here is to use the relation [26]

$$
\frac{d}{d t} \hat{r}=\frac{1}{r^{2}}(\mathbf{L} \times \hat{\mathbf{r}}) .
$$

Therefore, from

$$
\frac{d}{d t}(\mathbf{L} \times \mathbf{p})=\frac{d \mathbf{L}}{d t} \times \mathbf{p}+\mathbf{L} \times \frac{d \mathbf{p}}{d t},
$$

and with the help of the relations (20) and (22), we obtain

$$
\frac{d}{d t}(\mathbf{L} \times \mathbf{p})=\left(\boldsymbol{\nabla} V_{e x t} \times \mathbf{r}\right) \times \mathbf{p}+\boldsymbol{\nabla} V_{e x t} \times(\mathbf{r} \times \mathbf{p})-\frac{Z}{r^{2}}(\mathbf{L} \times \hat{r}) .
$$

On the other hand, taking the Runge-Lenz (RL) vector

$$
\mathbf{A}=\frac{1}{Z}(\mathbf{L} \times \mathbf{p})+\hat{\mathbf{r}}
$$

deriving it in time,

$$
\frac{d \mathbf{A}}{d t}=\frac{1}{Z} \frac{d}{d t}(\mathbf{L} \times \mathbf{p})+\frac{d}{d t} \hat{r}
$$

and using relations (23) and (25), we arrive at

$$
\frac{d \mathbf{A}}{d t}=\frac{1}{Z}\left[\left(\boldsymbol{\nabla} V_{e x t} \times \mathbf{r}\right) \times \mathbf{p}+\boldsymbol{\nabla} V_{e x t} \times(\mathbf{r} \times \mathbf{p})\right] .
$$

In the case of a hydrogen atom without external fields, i.e., $V_{\text {ext }}=0$, the right hand side of Eq. (28) is equal to zero, and the RL vector $\mathbf{A}$ is constant in time. The derivation of the RL vector for the Kepler motion without external fields was done long ago [27-29].

Our next goal is to integrate in time the right hand side of Eq. (28) in order to find the integrals of motion. Integrating Eq. (28) we find integrals which are linear functions of the $\mathrm{RL}$ vector. It is also possible to derive an equation analogous to Eq. (28) and yielding integrals of motion which are quadratic functions of the RL vector. This is done observing that

$$
\frac{d}{d t} \mathbf{A}^{2}=2 \mathbf{A} \cdot \frac{d \mathbf{A}}{d t}=\mathbf{A} \cdot \frac{2}{Z}\left[\left(\nabla V_{e x t} \times \mathbf{r}\right) \times \mathbf{p}+\nabla V_{e x t} \times(\mathbf{r} \times \mathbf{p})\right] .
$$

Note that Eqs. (28) and (29) are very general expressions which may be used to find integrals of motion for the Kepler problem, perturbed not only by the potential $V_{e x t}$ considered in this paper but also for any time-independent external potential $V_{\text {ext }}$. Furthermore, approximated integrals of motion can be also obtained by integrating Eqs. (28) and (29) over one cycle of the unperturbed Kepler motion as done, for example, in Ref. [30]. In the remainder of this section we derive classic integrals of motion, linear and quadratic in the parameters characterizing field strengths.

\section{A. Linear integrals}

The right hand side of Eq. (28) can be written in many different forms. Using well-known rules of vector algebra, it is not difficult to see that

$$
\begin{aligned}
\frac{d \mathbf{A}}{d t}= & \frac{1}{Z}\left[2\left(\mathbf{p} \cdot \boldsymbol{\nabla} V_{e x t}\right) \cdot \mathbf{r}-(\mathbf{r} \cdot \mathbf{p}) \cdot \boldsymbol{\nabla} V_{e x t}\right. \\
& \left.-\left(\mathbf{r} \cdot \boldsymbol{\nabla} V_{e x t}\right) \cdot \mathbf{p}\right],
\end{aligned}
$$

with components

$$
\begin{aligned}
\frac{d A_{q_{1}}}{d t}= & \frac{2 \gamma}{Z}\left[q_{2}\left(q_{1} p_{q_{2}}-q_{2} p_{q_{1}}\right)-q_{1} q_{3} p_{q_{3}}+\beta q_{3}\left(2 q_{1} p_{q_{3}}\right.\right. \\
& \left.\left.-q_{3} p_{q_{1}}\right)\right]+\frac{F}{Z}\left(2 q_{1} p_{q_{3}}-q_{3} p_{q_{1}}\right),
\end{aligned}
$$

$$
\begin{aligned}
\frac{d A_{q_{2}}}{d t}= & \frac{2 \gamma}{Z}\left[q_{1}\left(q_{2} p_{q_{1}}-q_{1} p_{q_{2}}\right)-q_{2} q_{3} p_{q_{3}}\right. \\
& \left.+\beta q_{3}\left(2 q_{2} p_{q_{3}}-q_{3} p_{q_{2}}\right)\right]+\frac{F}{Z}\left(2 q_{2} p_{q_{3}}-q_{3} p_{q_{2}}\right),
\end{aligned}
$$

$$
\begin{aligned}
& \frac{d}{d t}\left\{A_{q_{3}}+\frac{F}{2 Z}\left(q_{1}^{2}+q_{2}^{2}\right)\right\} \\
& =\frac{2 \gamma}{Z}\left[q_{1}\left(2 q_{3} p_{q_{1}}-q_{1} p_{q_{3}}\right) q_{2}\left(2 q_{3} p_{q_{2}}-q_{2} p_{q_{3}}\right)-\beta q_{3}\left(q_{1} p_{q_{1}}\right.\right. \\
& \left.\left.\quad+q_{2} p_{q_{2}}\right)\right] .
\end{aligned}
$$

In Eq. (33) we have already integrated the term proportional to the electric field intensity $F$, transposing it to the left hand side. The Stark term is always "integrable" and, therefore, it is always possible to find the integral of motion in the $q_{3}$ component. For the pure Stark effect $[\gamma=0$ in Eq. (33)], the integral is

$$
A_{q_{3}}+\frac{F}{2 Z}\left(q_{1}^{2}+q_{2}^{2}\right)
$$


a component of a sort of "generalized" Runge-Lenz vector already considered in an almost forgotten paper by Redmond [26].

The important question here is, how can we integrate Eqs. (31), (32) and (33)? We now show that several cases predicted to be integrable by the Painlevé analysis are indeed integrable. This is done by determining explicitly the corresponding integrals.

(a) $\beta=1(B=3 A, A=\gamma / 4)$ : Writing Eq. (33) as a function of the angular momentum, we obtain

$$
\frac{d}{d t}\left\{A_{q_{3}}+\frac{F}{2 Z}\left(q_{1}^{2}+q_{2}^{2}\right)\right\}=\frac{2 \gamma}{Z}\left(q_{1} L_{q_{2}}-q_{2} L_{q_{1}}\right) .
$$

Now, since

$$
\begin{gathered}
\frac{d}{d t} L_{q_{1}}^{2}=2 L_{q_{1}} \frac{d}{d t} L_{q_{1}}=-2 F q_{2} L_{q_{1}}, \\
\frac{d}{d t} L_{q_{2}}^{2}=2 L_{q_{2}} \frac{d}{d t} L_{q_{2}}=2 F q_{1} L_{q_{2}},
\end{gathered}
$$

we integrate the second term on the right-hand side of Eq. (35) to obtain

$$
\frac{d}{d t}\left\{A_{q_{3}}+\frac{F}{2 Z}\left(q_{1}^{2}+q_{2}^{2}\right)-\frac{\gamma}{F Z}\left(L_{q_{1}}^{2}+L_{q_{2}}^{2}\right)\right\}=0 .
$$

From this bracket we subtract a term proportional to $L_{q_{3}}^{2}$ (i.e., a constant integral of motion), thereby obtaining

$$
\frac{d}{d t} \mathcal{C}_{q_{3}}=0
$$

where

$$
\mathcal{C}_{q_{3}}=A_{q_{3}}+\frac{F}{2 Z}\left(q_{1}^{2}+q_{2}^{2}\right)-\frac{\gamma}{F Z} \mathbf{L}^{2} .
$$

This integral of motion contains the component of the generalized RL vector [Eq. (34)] for the Stark problem, and includes a new van der Waals term, proportional to the magnetic field strength $\gamma$. For $F=0$ the last term is singular. From Eq. (22) one sees the well-known fact [20-22,31,32] that the components $L_{q_{1}}, L_{q_{2}}$, and $L_{q_{3}}$ are separately integrals of motion. For $\gamma=0$ we recover the result for the pure Stark problem [26].

Now we want to confirm the existence of the integral of motion [Eq. (40)] through the numerical investigation of Eqs. (7) and (8) for values of $\beta$ around $\beta=1$. This is done with the help of Poincare surfaces of section (PSS's) in phase space. For a fixed value of the energy $\epsilon$ of the fourdimensional phase space of Eq. (6), the motion is confined to a three-dimensional energy shell, and the PSS is a twodimensional slice in this three-dimensional energy surface. Most of the information of the classical motion is contained in the set of all intersections of a given trajectory with the PSS.
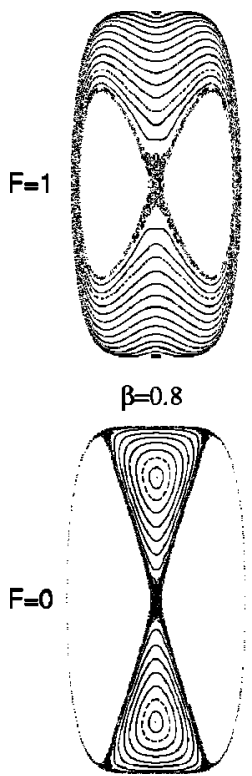

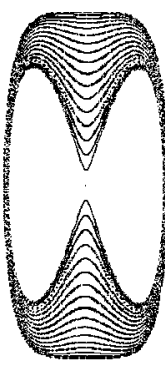

$\beta=1.0$

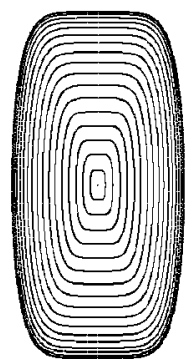

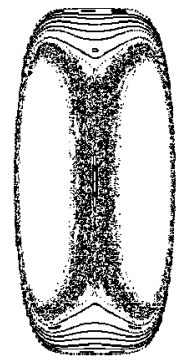

$\beta=1.2$

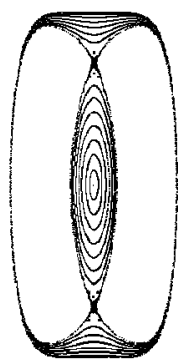

FIG. 1. Poincaré surfaces of section for $\beta=0.8,1.0$, and 1.2 (from left to right). The three surfaces on the top are calculated for $F=1$, and the surfaces on the bottom for $F=0$ (no electric field). See the text for more details.

Figure 1 shows the PSS for different values of $\beta$ and $F$. We plotted the coordinates $\left(u, p_{u}\right)$; therefore, the PSS is defined by all trajectories which intersect $v=0$ with $p_{v}>0$. The top (bottom) three surfaces of the figure show results for $F=1(F=0$, no electric field $)$ and different values of $\beta$. Results for $F=0$ are discussed below, in Sec. IV C. The values of $\gamma$ and $\epsilon$ are 6.0 and 0.0 , respectively, which means that we have very strong magnetic field and high energies. The reason for using strong values is because for those values of $\beta$ for which the system is nonintegrable we expect to have strong chaos, and therefore we can more clearly analyze the transition from regular to chaotic motion.

For $\beta=1$ and $F=1$ (top, center) the surface of Fig. 1 shows a regular structure. One elliptic fixed point at the center of the surface $\left(u=p_{u}=0\right)$ is surrounded by regular motion. For $\beta=0.8$ (top, left), just below the integrable case, the elliptic fix point is now a hyperbolic fix point and some irregular motion around it can be recognized. For $\beta=1.2$ (top, right), above the integrable case, the motion is mostly irregular. These numerical results clearly corroborate the existence of an integral of motion for $\beta=1, F=1$.

(b) $\beta=4(B=0, A=\gamma)$ : In this case Eq. (33) is

$$
\begin{aligned}
& \frac{d}{d t}\left\{A_{q_{3}}+\frac{F}{2 Z}\left(q_{1}^{2}+q_{2}^{2}\right)\right\} \\
& \quad=\frac{2 \gamma}{Z}\left[-2 q_{3}\left(q_{1} p_{q_{1}}+q_{2} p_{q_{2}}\right)-p_{q_{3}}\left(q_{1}^{2}+q_{2}^{2}\right)\right],
\end{aligned}
$$

and can be readily integrated,

$$
\frac{d}{d t}\left\{A_{q_{3}}+\frac{F}{2 Z}\left(q_{1}^{2}+q_{2}^{2}\right)+\frac{2 \gamma}{Z} q_{3}\left(q_{1}^{2}+q_{2}^{2}\right)\right\}=0,
$$

yielding the invariant 

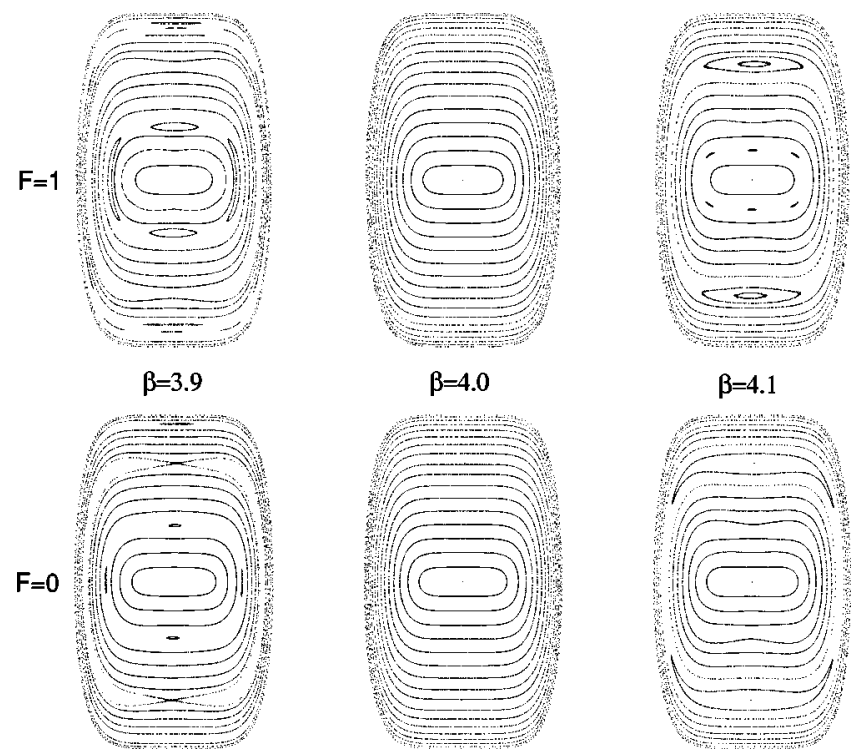

FIG. 2. Poincaré surfaces of section for $\beta=3.9,4.0$, and 4.1 (from left to right). The three surfaces on the top are calculated for $F=1$, and the surfaces on the bottom for $F=0$ (no electric field). See the text for additional details.

$$
G_{q_{3}}=A_{q_{3}}+\left[\frac{F}{2 Z}+\frac{2 \gamma}{Z} q_{3}\right]\left(q_{1}^{2}+q_{2}^{2}\right)
$$

This integral of motion is again a generalized RL integral which for $\gamma=0$ reduces to the result found by Redmond [26] for the Stark effect.

The integral of motion [Eq. (42)] can also be corroborated numerically as shown in Fig. 2. This figure shows the PSS for values of $\beta$ near the integrable case $\beta=4$. In the PSS at the center of the top $F=1$ row, we see that the motion is very regular having just a single fix point at $u=p_{u}=0$. For values of $\beta=3.9$ (left) and $\beta=4.1$ (right) we see the appearance of hyperbolic fix points which act to destroy the regular motion.

For $F=0$ (no electric field), Fig. 2 (center, bottom row) shows that we still have a regular motion. This is related with the fact, that for $F=0$, Eq. (42) reduces to

$$
G_{q_{3}}=A_{q_{3}}+\frac{2 \gamma}{Z} q_{3}\left(q_{1}^{2}+q_{2}^{2}\right)
$$

reproducing the known integral of motion for a trapped ion [31]. For $\beta=3.9$ (left) and $\beta=4.1$ (right) we see once again the appearance of some hyperbolic fix points which disrupt the regular motion. Note, for example, the appearance of six hyperbolic fix points near $u=p_{u}=0$ on the PSS at the top, right.

(c) $\beta=1 / 4 \quad(B=15 A, A=\gamma / 16)$ : This is the last possibly integrable candidate predicted by the Painlevé analysis. Rewriting Eqs. (31) and (32) as

$$
\begin{aligned}
\frac{d A_{q_{1}}}{d t}= & \frac{2 \gamma}{Z}\left[q_{2}\left(q_{1} p_{q_{2}}-q_{2} p_{q_{1}}\right)-\frac{1}{2}\left(q_{1} q_{3} p_{q_{3}}+\frac{q_{3}^{2}}{2} p_{q_{1}}\right)\right] \\
& +\frac{F}{Z}\left(2 q_{1} p_{q_{3}}-q_{3} p_{q_{1}}\right), \\
\frac{d A_{q_{2}}}{d t}= & \frac{2 \gamma}{Z}\left[-q_{1}\left(q_{1} p_{q_{2}}-q_{2} p_{q_{1}}\right)-\frac{1}{2}\left(q_{2} q_{3} p_{q_{3}}+\frac{q_{3}^{2}}{2} p_{q_{2}}\right)\right] \\
& +\frac{F}{Z}\left(2 q_{2} p_{q_{3}}-q_{3} p_{q_{2}}\right),
\end{aligned}
$$

we see that, for both equations, the term in parentheses which multiplies $\frac{1}{2}$ can be easily integrated.

For arbitrary values of $F$ we have not been able to find integrals, but for $F=0$ we obtain

$$
\begin{gathered}
\frac{d}{d t}\left\{A_{q_{1}}+\frac{\gamma}{2 Z} q_{1} q_{3}^{2}\right\}=\frac{2 \gamma}{Z}\left[q_{2}\left(q_{1} p_{q_{2}}-q_{2} p_{q_{1}}\right)\right], \\
\frac{d}{d t}\left\{A_{q_{2}}+\frac{\gamma}{2 Z} q_{2} q_{3}^{2}\right\}=\frac{2 \gamma}{Z}\left[-q_{1}\left(q_{1} p_{q_{2}}-q_{2} p_{q_{1}}\right)\right],
\end{gathered}
$$

which might also be written as

$$
\begin{gathered}
\frac{d}{d t}\left\{A_{q_{1}}+\frac{\gamma}{2 Z} q_{1} q_{3}^{2}\right\}=\frac{2 \gamma}{Z}\left(q_{2} L_{q_{3}}\right), \\
\frac{d}{d t}\left\{A_{q_{2}}+\frac{\gamma}{2 Z} q_{2} q_{3}^{2}\right\}=\frac{2 \gamma}{Z}\left(-q_{1} L_{q_{3}}\right) .
\end{gathered}
$$

These expressions are only integrable for $L_{q_{3}}=m=0$, yielding the generalized RL (GRL) components

$$
\begin{aligned}
& G_{q_{1}}=A_{q_{1}}+\frac{\gamma}{2 Z} q_{1} q_{3}^{2}, \\
& G_{q_{2}}=A_{q_{2}}+\frac{\gamma}{2 Z} q_{2} q_{3}^{2},
\end{aligned}
$$

which are the integrals of motion for $m=0$. Observe that for $m=0$ we have $t w o$ additional integrals of motion.

Figure 3 shows the PSS for the integrable case $\beta=1 / 4$ $=0.25$ and $F=0$ (bottom row, center). As expected, the motion is regular, and shows two elliptic fixed points on the upper and lower parts of the surface, both surrounded by ellipses. The surface for $\beta=0.2$ (bottom, left) shows what happens if we slightly change the value of $\beta$ from the value where the system is integrable. There is no longer any regular motion, and the trajectories fill most of the phase space densely. Some hyperbolic fixed points (or separatrices) can be observed; for example, near $u=p_{u}=0$ there are ten isolated island surrounded by ten hyperbolic points. The same behavior can be observed for $\beta=0.3$ (bottom, right). In this case we still see some localized remnants of the regular motion near the two original elliptic fixed points, but in general the motion is rather irregular. The three surfaces on the top of Fig. 3 show what happens if we add an electric field ( $F$ 

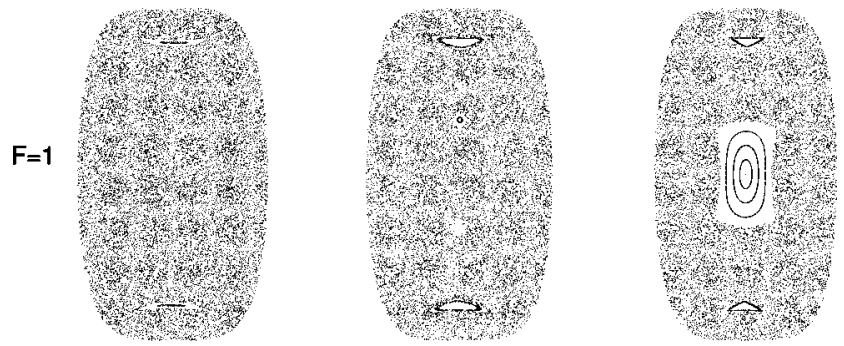

$\beta=0.2$

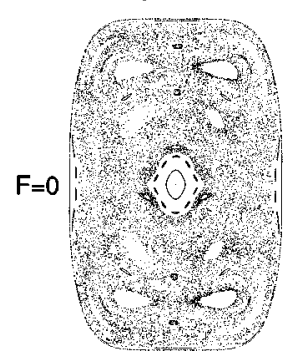

$\beta=1 / 4=0.25$

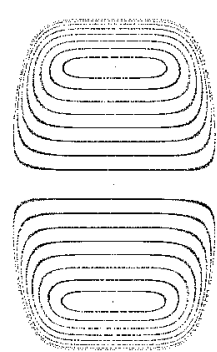

$\beta=0.3$

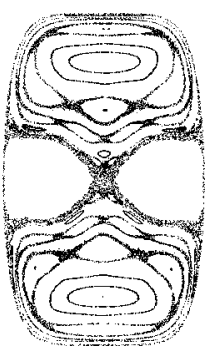

FIG. 3. Poincaré surfaces of section for $\beta=0.2,1 / 4$, and 0.3 (from left to right). The three surfaces on the top are calculated for $F=1$, and the surfaces on the bottom for $F=0$ (no electric field). See the text for more details.

$=1)$ to the problem: the system is no longer integrable, even when $\beta=1 / 4$. This is also the reason why we could not find any integral of motion for $F \neq 0$ and $\beta=1 / 4$ (see Table I). All three surfaces show a strong irregular motion.

\section{B. Generalized Runge-Lenz vector}

The above results show clearly the existence of a quite general Runge-Lenz-like vector for atomic problems subjected to the combined external fields defined in Eq. (2). Its components define integrals of motion for all three aforementioned cases. This vector may be conveniently written as

$$
\mathbf{G}=\mathbf{A}-\frac{2 \gamma}{3 Z}[(\mathbf{r} \times \mathbf{W}) \times \mathbf{r}]-\frac{F}{2 Z}[(\mathbf{r} \times \mathbf{S}) \times \mathbf{r}],
$$

where $\mathbf{W}=q_{1} \hat{\mathbf{i}}+q_{2} \hat{\mathbf{j}}+\beta q_{3} \hat{\mathbf{k}}$ and $\mathbf{S}=F \hat{\mathbf{k}}$ are the derivatives of the potential due to the van der Waals interaction and the

TABLE I. Values of $\beta$ for the integrable cases where the components of the GRL vector [Eq. (52)] are integrals of motion.

\begin{tabular}{ccccc}
\hline \hline$\beta$ & $\gamma$ & $F$ & $L_{q_{3}}=m$ & Classical integrals \\
\hline 1 & arb. & 0 & arb. & $\mathbf{G}^{2}+\frac{2 \gamma}{Z^{2}} r^{2} \mathbf{L}^{2}$ \\
4 & arb. & arb. & arb. & $G_{q_{3}}$ \\
4 & arb. & 0 & arb. & $G_{q_{3}}$ \\
$1 / 4$ & arb. & 0 & arb. & $G_{q_{1}}^{2}+G_{q_{2}}^{2} \frac{2 \gamma}{Z^{2}} r^{2} L_{q_{3}}^{2}$ \\
$1 / 4$ & arb. & 0 & 0 & $G_{q_{1}}, G_{q_{2}}$ \\
\hline \hline
\end{tabular}

electric field, respectively. The GRL vector [Eq. (52)] is a generalization to the Zeeman and van der Waals interactions of the pure Stark vector obtained previously by Redmond [26].

The components of Eq. (52) are

$$
\begin{gathered}
G_{q_{1}}=A_{q_{1}}+\frac{2 \gamma}{3 Z}(1-\beta) q_{1} q_{3}^{2}-\frac{F}{2 Z} q_{1} q_{3}, \\
G_{q_{2}}=A_{q_{2}}+\frac{2 \gamma}{3 Z}(1-\beta) q_{2} q_{3}^{2}-\frac{F}{2 Z} q_{2} q_{3}, \\
G_{q_{3}}=A_{q_{3}}-\frac{2 \gamma}{3 Z}(1-\beta) q_{3}\left(q_{1}^{2}+q_{2}^{2}\right)+\frac{F}{2 Z}\left(q_{1}^{2}+q_{2}^{2}\right),
\end{gathered}
$$

and do not represent integrals of motion in general, for arbitrary values of the fields. These components are integrals of motion only for the specific situations discussed in detail above.

Note that for $\beta=1, F \neq 0$ and arbitrary $\gamma$, the GRL vector does not reproduce integral (40). We believe that integral (40) takes into account a special symmetry which cannot be obtained from the GRL vector. We were not able to find a vector from which one may obtain integral (40). Apart from this "singular" case, all other integrals found can be obtained directly from the GRL vector [Eq. (52)]. The GRL vector will be very useful in Sec. IV C.

\section{Quadratic integrals}

The procedure to obtain the integrals for the quadratic case is analogous to that for the linear case, and we limit ourselves to presenting only the most important final equations. Contributions which are quadratic functions of the field-strength parameters arise from terms proportional to the square of the GRL vector [see Eq. (29)]. If the square of the GRL vector was itself an integral of motion, then the following scalar would be identically zero:

$$
\frac{1}{2} \frac{d}{d t} \mathbf{G}^{2}=\mathbf{G} \cdot \frac{d \mathbf{G}}{d t}=G_{q_{1}} \frac{d G_{q_{1}}}{d t}+G_{q_{2}} \frac{d G_{q_{2}}}{d t}+G_{q_{3}} \frac{d G_{q_{3}}}{d t} .
$$

Motivated by the expression above, we look for integrals of motion which, while depending on the same individual terms as the scalar above, are more general. A simple way of doing this is given by the Ansatz

$$
\zeta=c_{1} G_{q_{1}} \frac{d G_{q_{1}}}{d t}+c_{2} G_{q_{2}} \frac{d G_{q_{2}}}{d t}+c_{3} G_{q_{3}} \frac{d G_{q_{3}}}{d t},
$$

where $c_{i}, i=1,2$, and 3 , are constants to be determined. In Eq. (57) we use the GRL vector instead of the RL vector of Eq. (29). This simplifies considerably the calculations. In what follows we consider only integrals which are not the square of the integrals already found for the linear cases. 
Table I presents a summary of the values of $\beta$ for which the components of the GRL vector [Eq. (52)] are integrals of motion.

(a) $\beta=1 \quad(B=3 A, A=\gamma / 4)$ : For this combination of parameters we could not find additional integrals of motion when $F \neq 0$ and, consequently, we consider $F=0$ only. From Eq. (22) we know that $L_{q_{1}}, L_{q_{2}}$, and $L_{q_{3}}$ are integrals of motion, while Eq. (40) is no longer an integral for $F=0$. For $c_{i}=1, i=1,2$, and 3, Eq. (57) may be rearranged to yield

$$
\begin{aligned}
& \frac{d}{d t}\left(G_{q_{1}}^{2}+G_{q_{2}}^{2}+G_{q_{3}}^{2}\right) \\
& \quad=-\frac{4 \gamma}{Z^{2}}\left(q_{1} p_{q_{1}}+q_{2} p_{q_{2}}+q_{3} p_{q_{3}}\right)\left(L_{q_{1}}^{2}+L_{q_{2}}^{2}+L_{q_{3}}^{2}\right) .
\end{aligned}
$$

The right hand side of this equation can be integrated, observing that

$$
\frac{d r}{d t}=\frac{1}{r}\left(q_{1} p_{q_{1}}+q_{2} p_{q_{2}}+q_{3} p_{q_{3}}\right),
$$

and that, consequently, the integral of motion has the form

$$
I_{1}^{S}=G_{q_{1}}^{2}+G_{q_{2}}^{2}+G_{q_{3}}^{2}+\frac{2 \gamma}{Z^{2}} r^{2} \mathbf{L}^{2} .
$$

Note that now we have a total of five integrals of motion, $H, L_{q_{1}}, L_{q_{2}}, L_{q_{3}}$, and $I_{1}^{S}$, a result telling us that the system is super-integrable [33] because there are more integrals of motion than degrees of freedom.

(b) $\beta=4 \quad(B=0, A=\gamma)$ : For this set of parameters we could not find additional integrals of motion.

(c) $\beta=1 / 4 \quad(B=15 A, A=\gamma / 16)$ : Here we take $c_{1}=c_{2}$ $=1$ and $c_{3}=0$ obtaining for Eq. (57) the simplified expression

$$
\frac{d}{d t}\left(G_{q_{1}}^{2}+G_{q_{2}}^{2}\right)=-\frac{4 \gamma}{Z^{2}}\left(q_{1} p_{q_{1}}+q_{2} p_{q_{2}}+q_{3} p_{q_{3}}\right) L_{q_{3}}^{2} .
$$

Using Eq. (58) once again, we obtain the integral

$$
I_{2}^{S}=G_{q_{1}}^{2}+G_{q_{2}}^{2}+\frac{2 \gamma}{Z^{2}} r^{2} L_{q_{3}}^{2},
$$

which is a particular case of integral (59). This integral corresponds to that found for the motion of a trapped ion [31].

\section{QUANTUM OPERATORS}

In this section we derive the quantum operators which correspond to the classical integrals of motion obtained above. The methodology used in Sec. IV also works mutatis mutandis for operators. After suitable symmetrization, the quantum version of the time derivative of the RL vector is

$$
\begin{aligned}
\frac{d \hat{\mathbf{A}}}{d t}= & \frac{1}{2 Z}\left[\left(\hat{\nabla} V_{e x t} \times \hat{\mathbf{r}}\right) \times \hat{\mathbf{p}}-\hat{\mathbf{p}} \times\left(\hat{\nabla} V_{e x t} \times \hat{\mathbf{r}}\right)\right. \\
& \left.+\hat{\boldsymbol{\nabla}} V_{e x t} \times(\hat{\mathbf{r}} \times \hat{\mathbf{p}})-(\hat{\mathbf{r}} \times \hat{\mathbf{p}}) \times \hat{\boldsymbol{\nabla}} V_{e x t}\right],
\end{aligned}
$$

where

$$
\hat{\boldsymbol{\nabla}} V_{e x t}=\partial_{x} V_{e x t} \hat{\mathbf{i}}+\partial_{y} V_{e x t} \hat{\mathbf{j}}+\partial_{z} V_{e x t} \hat{\mathbf{k}}
$$

Following the same procedure used in Sec. IV, for the components of the RL operator we obtain

$$
\begin{aligned}
\frac{d \hat{A}_{\hat{q}_{1}}}{d t}= & \frac{2 \gamma}{Z}\left\{\hat{q}_{2}\left(\hat{q}_{1} p_{\hat{q}_{2}}-\hat{q}_{2} p_{\hat{q}_{1}}\right)-\hat{q}_{1} \hat{q}_{3} p_{\hat{q}_{3}}\right. \\
& \left.+\beta\left[\hat{q}_{3}\left(2 \hat{q}_{1} p_{\hat{q}_{3}}-\hat{q}_{3} p_{\hat{q}_{1}}\right)-i \hbar \hat{q}_{1}\right]\right\} \\
& +\frac{F}{Z}\left(2 \hat{q}_{1} \hat{p}_{\hat{q}_{3}}-\hat{q}_{3} \hat{p}_{\hat{q}_{1}}\right), \\
\frac{d \hat{A}_{\hat{q}_{2}}}{d t}= & \frac{2 \gamma}{Z}\left\{\hat{q}_{1}\left(\hat{q}_{2} p_{\hat{q}_{1}}-\hat{q}_{1} p_{\hat{q}_{2}}\right)-\hat{q}_{2} \hat{q}_{3} p_{\hat{q}_{3}}\right. \\
& \left.+\beta\left[\hat{q}_{3}\left(2 \hat{q}_{2} p_{\hat{q}_{3}}-\hat{q}_{3} p_{\hat{q}_{2}}\right)-i \hbar \hat{q}_{2}\right]\right\} \\
& +\frac{F}{Z}\left(2 \hat{q}_{2} \hat{p}_{\hat{q}_{3}}-\hat{q}_{3} \hat{p}_{\hat{q}_{2}}\right),
\end{aligned}
$$

$$
\begin{aligned}
\frac{d \hat{A}_{\hat{q}_{3}}}{d t}= & \frac{2 \gamma}{Z}\left\{\hat{q}_{1}\left(2 \hat{q}_{3} p_{\hat{q}_{1}}-\hat{q}_{1} p_{\hat{q}_{3}}\right)+\hat{q}_{2}\left(2 \hat{q}_{3} p_{\hat{q}_{2}}-\hat{q}_{2} p_{\hat{q}_{3}}\right)\right. \\
& \left.-\beta \hat{q}_{3}\left(\hat{q}_{1} p_{\hat{q}_{1}}+\hat{q}_{2} p_{\hat{q}_{2}}\right)\right\}+\frac{F}{Z}\left(i \hbar-\hat{q}_{1} \hat{p}_{\hat{q}_{1}}-\hat{q}_{2} \hat{p}_{\hat{q}_{2}}\right),
\end{aligned}
$$

where this last equation may be rewritten as

$$
\begin{aligned}
\frac{d}{d t}\left\{\hat{A}_{\hat{q}_{3}}+\frac{F}{2 Z}\left(\hat{q}_{1}^{2}+\hat{q}_{2}^{2}\right)\right\} \\
=\frac{2 \gamma}{Z}\left\{\hat{q}_{1}\left(2 \hat{q}_{3} p_{\hat{q}_{1}}-\hat{q}_{1} p_{\hat{q}_{3}}\right)+\hat{q}_{2}\left(2 \hat{q}_{3} p_{\hat{q}_{2}}-\hat{q}_{2} p_{\hat{q}_{3}}\right)\right. \\
\left.\quad-\beta \hat{q}_{3}\left(\hat{q}_{1} p_{\hat{q}_{1}}+\hat{q}_{2} p_{\hat{q}_{2}}\right)\right\} .
\end{aligned}
$$

Comparing these equations with the corresponding classical ones in Eqs. (31), (32), and (33), one sees that the quantum operators contain quantum corrections proportional to $\hbar$.

The equations above cannot be integrated in general. However, for the situations passing the three steps of the Painlevé analysis we may proceed as follows.

\section{A. Linear operators}

Similarly to the classical case, we start by deriving the linear quantum operators for cases (a), (b), and (c). 
(a) $\beta=1 \quad(B=3 A, A=\gamma / 4)$ : Here the only possibly integrable component is that of Eq. (67):

$$
\frac{d}{d t}\left\{\hat{A}_{\hat{q}_{3}}+\frac{F}{2 Z}\left(\hat{q}_{1}^{2}+\hat{q}_{2}^{2}\right)\right\}=\frac{2 \gamma}{Z}\left(\hat{q}_{1} \hat{L}_{\hat{q}_{2}}-i \hbar \hat{q}_{3}-\hat{q}_{2} L_{\hat{q}_{1}}\right) .
$$

The right hand side can be integrated, observing that

$$
\begin{aligned}
\frac{d}{d t}\left(\hat{L}_{1}^{2}+\hat{L}_{2}^{2}\right) & =\frac{d \hat{L}_{1}}{d t} \hat{L}_{1}+\hat{L}_{1} \frac{d \hat{L}_{1}}{d t}+\frac{d \hat{L}_{2}}{d t} \hat{L}_{2}+\hat{L}_{2} \frac{d \hat{L}_{2}}{d t} \\
& =F\left(-\hat{q}_{2} \hat{L}_{\hat{q}_{1}}-\hat{L}_{\hat{q}_{1}} \hat{q}_{2}+\hat{q}_{1} \hat{L}_{\hat{q}_{2}}+\hat{L}_{\hat{q}_{2}} \hat{q}_{1}\right) \\
& =2 F\left(\hat{q}_{1} \hat{L}_{\hat{q}_{2}}-\hat{q}_{2} \hat{L}_{\hat{q}_{1}}-i \hbar \hat{q}_{3}\right),
\end{aligned}
$$

which yields

$$
\frac{d}{d t}\left\{\hat{A}_{\hat{q}_{3}}+\frac{F}{2 Z}\left(\hat{q}_{1}^{2}+\hat{q}_{2}^{2}\right)-\frac{\gamma}{F Z}\left(\hat{L}_{\hat{q}_{1}}^{2}+\hat{L}_{\hat{q}_{2}}^{2}\right)\right\}=0 .
$$

Therefore, the quantum operator has the form

$$
\hat{\mathcal{C}}_{\hat{q}_{3}}=\hat{A}_{\hat{q}_{3}}+\frac{F}{2 Z}\left(\hat{q}_{1}^{2}+\hat{q}_{2}^{2}\right)-\frac{\gamma}{F Z} \hat{\mathbf{L}}^{2}
$$

analogous to its classical counterpart [Eq. (40)].

(b) $\beta=4 \quad(B=0, A=\gamma)$ : Once again, we use Eq. (67), which now reads

$$
\begin{aligned}
\frac{d}{d t}\left\{\hat{A}_{\hat{q}_{3}}+\frac{F}{2 Z}\left(\hat{q}_{1}^{2}+\hat{q}_{2}^{2}\right)\right\}= & \frac{2 \gamma}{Z}\left[-2 \hat{q}_{3}\left(-i \hbar+\hat{q}_{1} \hat{p}_{\hat{q}_{1}}+\hat{q}_{2} \hat{p}_{\hat{q}_{2}}\right)\right. \\
& \left.-\left(\hat{q}_{1}^{2}+\hat{q}_{2}^{2}\right) \hat{p}_{\hat{q}_{3}}\right] .
\end{aligned}
$$

Then, with the help of

$$
\begin{aligned}
\frac{d}{d t}\left[\hat{q}_{3}\left(\hat{q}_{1}^{2}+\hat{q}_{2}^{2}\right)\right]= & \frac{d \hat{q}_{3}}{d t}\left(\hat{q}_{1}^{2}+\hat{q}_{2}^{2}\right)+\hat{q}_{3} \frac{d\left(\hat{q}_{1}^{2}+\hat{q}_{2}^{2}\right)}{d t}=\left(\hat{q}_{1}^{2}\right. \\
& \left.+\hat{q}_{2}^{2}\right) \hat{p}_{\hat{q}_{3}}+2 \hat{q}_{3}\left(-i \hbar+\hat{q}_{1} \hat{p}_{\hat{q}_{1}}+\hat{q}_{2} \hat{p}_{\hat{q}_{2}}\right),
\end{aligned}
$$

we may integrate Eq. (71), obtaining the operator

$$
\hat{G}_{\hat{q}_{3}}=\hat{A}_{\hat{q}_{3}}+\left[\frac{F}{2 Z}+\frac{2 \gamma}{Z} \hat{q}_{3}\right]\left(\hat{q}_{1}^{2}+\hat{q}_{2}^{2}\right),
$$

which mimics its classical counterpart [Eq. (42)].

(c) $\beta=1 / 4 \quad(B=15 A, A=\gamma / 16)$ : For these parameters it is possible to integrate Eqs. (64) and (65) for $F=0$, namely,

$$
\begin{aligned}
\frac{d \hat{A}_{\hat{q}_{1}}}{d t}= & \frac{2 \gamma}{Z}\left[-\frac{1}{2} \hat{q}_{1} \hat{q}_{3} \hat{p}_{\hat{q}_{3}}-\left(\hat{q}_{2}^{2}+\frac{1}{4} \hat{q}_{3}^{2}\right) \hat{p}_{\hat{q}_{1}}+\hat{q}_{1} \hat{q}_{2} \hat{p}_{\hat{q}_{2}}\right. \\
& \left.-\frac{1}{4} i \hbar \hat{q}_{1}\right],
\end{aligned}
$$

$$
\begin{aligned}
\frac{d \hat{A}_{\hat{q}_{2}}}{d t}= & \frac{2 \gamma}{Z}\left[-\frac{1}{2} \hat{q}_{2} \hat{q}_{3} \hat{p}_{\hat{q}_{3}}-\left(\hat{q}_{1}^{2}+\frac{1}{4} \hat{q}_{3}^{2}\right) \hat{p}_{\hat{q}_{2}}+\hat{q}_{1} \hat{q}_{2} \hat{p}_{\hat{q}_{1}}\right. \\
& \left.-\frac{1}{4} i \hbar \hat{q}_{2}\right],
\end{aligned}
$$

which may written more conveniently as

$$
\begin{aligned}
& \frac{d \hat{A}_{\hat{q}_{1}}}{d t}=\frac{\gamma}{2 Z}\left(-i \hbar \hat{q}_{1}-2 \hat{q}_{1} \hat{q}_{3} \hat{p}_{\hat{q}_{3}}-\hat{q}_{3}^{2} \hat{p}_{\hat{q}_{1}}+4 \hat{q}_{2} \hat{L}_{\hat{q}_{3}}\right), \\
& \frac{d \hat{A}_{\hat{q}_{2}}}{d t}=\frac{\gamma}{2 Z}\left(-i \hbar \hat{q}_{2}-2 \hat{q}_{2} \hat{q}_{3} \hat{p}_{\hat{q}_{3}}-\hat{q}_{3}^{2} \hat{p}_{\hat{q}_{2}}-4 \hat{q}_{1} \hat{L}_{\hat{q}_{3}}\right) .
\end{aligned}
$$

From the fact that

$$
\frac{d}{d t}\left(\hat{q}_{1} \hat{q}_{3}^{2}\right)=\frac{d \hat{q}_{1}}{d t} \hat{q}_{3}^{2}+\hat{q}_{1} \frac{d \hat{q}_{3}^{2}}{d t}=\hat{q}_{3}^{2} \hat{p}_{\hat{q}_{1}}+2 \hat{q}_{1} \hat{q}_{3} \hat{p}_{\hat{q}_{3}}-i \hbar \hat{q}_{1},
$$

we find the relations

$$
\begin{aligned}
& -2 \hat{q}_{1} \hat{q}_{3} \hat{p}_{\hat{q}_{3}}-\hat{q}_{3}^{2} \hat{p}_{\hat{q}_{1}}=-\frac{d}{d t}\left(\hat{q}_{1} \hat{q}_{3}^{2}\right)-i \hbar \hat{q}_{1}, \\
& -2 \hat{q}_{2} \hat{q}_{3} \hat{p}_{\hat{q}_{3}}-\hat{q}_{3}^{2} \hat{p}_{\hat{q}_{2}}=-\frac{d}{d t}\left(\hat{q}_{2} \hat{q}_{3}^{2}\right)-i \hbar \hat{q}_{2} .
\end{aligned}
$$

which, substituted into Eqs. (73) and (74), yield

$$
\begin{aligned}
& \frac{d}{d t}\left\{\hat{A}_{\hat{q}_{1}}+\frac{\gamma}{2 Z} \hat{q}_{1} \hat{q}_{3}^{2}\right\}=\frac{\gamma}{Z}\left(-i \hbar \hat{q}_{1}+2 \hat{q}_{2} \hat{L}_{\hat{q}_{3}}\right), \\
& \frac{d}{d t}\left\{\hat{A}_{\hat{q}_{2}}+\frac{\gamma}{2 Z} \hat{q}_{2} \hat{q}_{3}^{2}\right\}=\frac{\gamma}{Z}\left(-i \hbar \hat{q}_{2}-2 \hat{q}_{1} \hat{L}_{\hat{q}_{3}}\right) .
\end{aligned}
$$

When compared with their classical counterparts, Eqs. (48) and (49), the quantum equations are seen to contain one extra term on the right hand side. With the help of the familiar commutation relations

$$
\begin{gathered}
{\left[\hat{L}_{\hat{q}_{3}}, \hat{q}_{1}\right]=i \hbar \hat{q}_{2},} \\
{\left[\hat{L}_{\hat{q}_{3}}, \hat{q}_{2}\right]=-i \hbar \hat{q}_{1},}
\end{gathered}
$$

we rewrite Eqs. (75) and (76) as

$$
\begin{gathered}
\frac{d}{d t}\left\{\hat{A}_{\hat{q}_{1}}+\frac{\gamma}{2 Z} \hat{q}_{1} \hat{q}_{3}^{2}\right\}=\frac{\gamma}{Z}\left(\hat{L}_{\hat{q}_{3}} \hat{q}_{2}+\hat{q}_{2} \hat{L}_{\hat{q}_{3}}\right), \\
\frac{d}{d t}\left\{\hat{A}_{\hat{q}_{2}}+\frac{\gamma}{2 Z} \hat{q}_{2} \hat{q}_{3}^{2}\right\}=-\frac{\gamma}{Z}\left(\hat{L}_{\hat{q}_{3}} \hat{q}_{1}+\hat{q}_{1} \hat{L}_{\hat{q}_{3}}\right),
\end{gathered}
$$

which for $\hat{L}_{\hat{q}_{3}}=m=0$ give the operators 


$$
\begin{aligned}
& \hat{G}_{\hat{q}_{1}}=\hat{A}_{\hat{q}_{1}}+\frac{\gamma}{2 Z} \hat{q}_{1} \hat{q}_{3}^{2}, \\
& \hat{G}_{\hat{q}_{2}}=\hat{A}_{\hat{q}_{2}}+\frac{\gamma}{2 Z} \hat{q}_{2} \hat{q}_{3}^{2},
\end{aligned}
$$

coincident with the classical integrals in Eqs. (50) and (51).

\section{B. Generalized Runge-Lenz operator}

As for the classical integrals, here it is also possible to define a convenient generalized operator from which, for suitable parameters, we might derive as particular cases all the operators discussed above. This generalized operator is defined by

$$
\hat{\mathbf{G}}=\hat{\mathbf{A}}-\frac{2}{3 Z}[(\hat{\mathbf{r}} \times \hat{\mathbf{W}}) \times \hat{\mathbf{r}}]-\frac{1}{2 Z}[(\hat{\mathbf{r}} \times \hat{\mathbf{S}}) \times \hat{\mathbf{r}}],
$$

where $\hat{\mathbf{W}}=\left(\hat{q}_{1} \hat{\mathbf{i}}+\hat{q}_{2} \hat{\mathbf{j}}+\beta \hat{q}_{3} \hat{\mathbf{k}}\right)$ and $\hat{\mathbf{S}}=F \hat{\mathbf{k}}$ are the operators of the potential due to van de Waals interaction and the electric field, respectively. The components of this operator are

$$
\begin{gathered}
\hat{G}_{\hat{q}_{1}}=\hat{A}_{\hat{q}_{1}}+\frac{2 \gamma}{3 Z}(1-\beta) \hat{q}_{1} \hat{q}_{3}^{2}-\frac{F}{2 Z} \hat{q}_{1} \hat{q}_{3}, \\
\hat{G}_{\hat{q}_{2}}=\hat{A}_{\hat{q}_{2}}+\frac{2 \gamma}{3 Z}(1-\beta) \hat{q}_{2} \hat{q}_{3}^{2}-\frac{F}{2 Z} \hat{q}_{2} \hat{q}_{3}, \\
\hat{G}_{\hat{q}_{3}}=\hat{A}_{\hat{q}_{3}}-\frac{2 \gamma}{3 Z}(1-\beta) \hat{q}_{3}\left(\hat{q}_{1}^{2}+\hat{q}_{2}^{2}\right)+\frac{F}{2 Z}\left(\hat{q}_{1}^{2}+\hat{q}_{2}^{2}\right) .
\end{gathered}
$$

In Sec. V C we use the above GRL operator to discuss operators which are quadratic functions of the field strength parameters.

\section{Quadratic operators}

The quantum operator corresponding to Eq. (57) is

$$
\frac{1}{2} \frac{d}{d t} \hat{\mathbf{G}}^{2}=\hat{\mathbf{G}} \cdot \frac{d \hat{\mathbf{G}}}{d t}+\frac{d \hat{\mathbf{G}}}{d t} \cdot \hat{\mathbf{G}}
$$

which motivates us to consider the extended form

$$
\begin{aligned}
\mathcal{Z}= & c_{1}\left(\hat{G}_{\hat{q}_{1}} \frac{d \hat{G}_{\hat{q}_{1}}}{d t}+\frac{d \hat{G}_{\hat{q}_{1}}}{d t} \hat{G}_{\hat{q}_{1}}\right)+c_{2}\left(\hat{G}_{\hat{q}_{2}} \frac{d \hat{G}_{\hat{q}_{2}}}{d t}+\frac{d \hat{G}_{\hat{q}_{2}}}{d t} \hat{G}_{\hat{q}_{2}}\right) \\
& +c_{3}\left(\hat{G}_{\hat{q}_{3}} \frac{d \hat{G}_{\hat{q}_{3}}}{d t}+\frac{d \hat{G}_{\hat{q}_{3}}}{d t} \hat{G}_{\hat{q}_{3}}\right),
\end{aligned}
$$

where $c_{i}$ are arbitrary constants. We now present the final results for the integrable cases.

(a) $\beta=1 \quad(B=3 A, A=\gamma / 4)$ : When $c_{i}=1, i=1,2$, and 3, we find the quantum operator

$$
\hat{I}_{1}^{S}=\hat{G}_{\hat{q}_{1}}^{2}+\hat{G}_{\hat{q}_{2}}^{2}+\hat{G}_{\hat{q}_{3}}^{2}+\frac{2 \gamma}{Z^{2}} \hat{r}^{2} \hat{\mathbf{L}}^{2}+\frac{\gamma \hbar^{2}}{Z^{2}} \hat{r}^{2},
$$

TABLE II. Values of $\beta$ for the integrable cases where the components of the GRL operator [Eq. (81)] are good quantum numbers.

\begin{tabular}{ccccc}
\hline \hline$\beta$ & $\gamma$ & $F$ & $L_{q_{3}}=m$ & Quantum operators \\
\hline 1 & arb. & 0 & arb. & $\hat{\mathbf{G}}^{2}+\frac{2 \gamma}{Z^{2}} \hat{r}^{2} \hat{\mathbf{L}}^{2}$ \\
4 & arb. & arb. & arb. & $\hat{G}_{q_{3}}$ \\
4 & arb. & 0 & arb. & $\hat{G}_{q_{3}}$ \\
$1 / 4$ & arb. & 0 & arb. & $\hat{G}_{q_{1}}^{2}+\hat{G}_{q_{2}}^{2} \frac{2 \gamma}{Z^{2}} \hat{r}^{2} \hat{L}_{q_{3}}^{2}$ \\
$1 / 4$ & arb. & 0 & 0 & $\hat{G}_{q_{1}}, \hat{G}_{q_{2}}$ \\
\hline \hline
\end{tabular}

which, when compared with the corresponding classical integral [Eq. (59)], is seen to have an additional quantum correction proportional to $\hbar^{2}$.

(b) $\beta=4 \quad(B=0, A=\gamma)$ : For this set of parameters we were not able to find additional operators.

(c) $\beta=1 / 4 \quad(B=15 A, A=\gamma / 16)$ : For $c_{1}=c_{2}=1$ and $c_{3}$ $=0$, we find

$$
\hat{I}_{2}^{S}=\hat{G}_{\hat{q}_{1}}^{2}+\hat{G}_{\hat{q}_{2}}^{2}+\frac{2 \gamma}{Z^{2}} r^{2} \hat{L}_{\hat{q}_{3}}^{2}+\frac{\gamma \hbar^{2}}{2 Z^{2}}\left(\hat{r}^{2}+\hat{q}_{3}^{2}\right) .
$$

Comparing this operator with Eq. (61), we see that, again, the operator has a quantum correction proportional to $\hbar^{2}$. Table II summarizes the values of $\beta$ for which the components of the GRL operator [Eq. (81)] are good quantum numbers.

\section{CONCLUSIONS}

We studied the classical and quantum integrability of the problem of hydrogenic atoms subjected to external parallel electric and magnetic fields and also to the van der Waals interaction, as defined by Eqs. (1) and (2). With the help of a Painlevé analysis we discovered five exact integrals of motion as well as eight exact quantum operators, which to our knowledge were never given in the literature before. Table III presents a summary of our findings. In this table, integrals (operators) which are written between brackets are thought to be new results. All integrals (operators), with exception of $\mathcal{C}_{q_{3}}\left(\hat{\mathcal{C}}_{q_{3}}\right)$ from Eq. (40) [Eq. (70)], were found to be the components of a generalized Runge-Lenz (GRL) vector (operator) (52) [Eq. (81)].

An additional result is that concerning the quantum corrections for the operators which are quadratic functions of the aforementioned GRL operator. All such corrections are invariably found to be proportional to $\hbar^{2}$, a term obviously absent from the corresponding classical integral limits (see Table III). Proportionality factors depending on $\hbar^{2}$ were previously found to occur for certain classes of duality transformations interconnecting integrable Hamiltonian systems [19]. To a given classical system, such duality transforma- 
TABLE III. Summary of all known integrable cases along with their associated classical integrals and quantum operators. Results inside curly brackets are thought to be new.

\begin{tabular}{|c|c|c|c|c|c|}
\hline$\beta$ & $\gamma$ & $F$ & $L_{q_{3}}=m$ & Classical integrals & Quantum operators \\
\hline 1 & arb. & arb. & arb. & $\left\{\mathcal{C}_{q_{3}}\right\}$ & $\left\{\hat{\mathcal{C}}_{\hat{q}_{3}}\right\}$ \\
\hline 1 & arb. & 0 & arb. & $L_{q_{1}}, L_{q_{2}},\left\{\mathbf{G}^{2}+\frac{2 \gamma}{Z^{2}} r^{2} \mathbf{L}^{2}\right\}$ & $\hat{L}_{\hat{q}_{1}}, \hat{L}_{\hat{q}_{2}},\left\{\hat{\mathbf{G}}^{2}+\frac{2 \gamma}{Z^{2}} \hat{r}^{2} \hat{\mathbf{L}}^{2}+\frac{\gamma \hbar^{2}}{Z^{2}} \hat{r}^{2}\right\}$ \\
\hline 4 & arb. & arb. & arb. & $\left\{G_{q_{3}}\right\}$ & $\left\{\hat{G}_{q_{3}}\right\}$ \\
\hline 4 & arb. & 0 & arb. & $G_{q_{3}}$ & $\left\{\hat{G}_{q_{3}}\right\}$ \\
\hline 0 & 0 & arb. & arb. & $G_{q_{3}}$ & $\hat{G}_{q_{3}}$ \\
\hline $1 / 4$ & arb. & 0 & arb. & $G_{q_{1}}^{2}+G_{q_{2}}^{2}+\frac{2 \gamma}{Z^{2}} r^{2} L_{q_{3}}^{2}$ & $\left\{\hat{G}_{q_{1}}^{2}+\hat{G}_{q_{2}}^{2}+\frac{2 \gamma}{Z^{2}} r^{2} \hat{L}_{\hat{q}_{3}}^{2}+\frac{\gamma \hbar^{2}}{2 Z^{2}}\left(\hat{r}^{2}+\hat{q}_{3}^{2}\right)\right\}$ \\
\hline $1 / 4$ & arb. & 0 & 0 & $\left\{G_{q_{1}}\right\},\left\{G_{q_{2}}\right\}$ & $\left\{\hat{G}_{q_{1}}\right\},\left\{\hat{G}_{q_{2}}\right\}$ \\
\hline
\end{tabular}

tions allow one to associate a quantum system which is not necessarily unique [19].

We believe the exact results reported in this paper to be helpful to understand the dynamics of more complicated classical and quantum systems. For example, the new integrals of motion can be used in the study of quantum chaos in atoms along the lines of what was done in, e.g., Refs. $[20,22,30,34]$ (and references therein). Another interesting problem is to investigate what happens to initially localized wave packets when one smoothly varies the relevant parameters away from the integrable limit. How much does the spreading and the speed of dispersion depend on the deviation from integrability, and so forth. In this respect, the interesting technique used in Ref. [16] could be of interest. Our integrals of motion may also be used to analyze the motion of an ion in a Paul trap $[31,32]$, to obtain a semiclassical formula to describe photoabsorption in atoms in external fields which have a regular spectrum [35], to understand fundamental questions in the semiclassical description of widthweighted spectra in atoms due to tunneling through a potential barrier [36], to analyze the interaction of atoms through van der Waals forces [37], and to investigate the effect of relaxing the perpendicular orientation of the electric and magnetic fields [38]. An interesting open problem is to repeat the present computations for the experimental setup discussed by Baumann [39], and for some variants of it.

\section{ACKNOWLEDGMENTS}

J.A.C.G. is grateful to PRAXIS XXI (Portugal) and CNPq (Brazil) for partial support.
[1] P. M. Koch and K. A. H. van Leeuwen, Phys. Rep. 255, 290 (1995).

[2] P. Schmelcher and W. Schweizer, Atoms and Molecules in Strong External Fields (Plenum Press, New York, 1998); H. Hasegawa, M. Robnik, and G. Wunner, Prog. Theor. Phys. Suppl. 98, 198 (1989).

[3] J. P. Santos, F. Mota-Furtado, M. F. Laranjeira, and F. Parente, Phys. Rev. A 59, 1703 (1999).

[4] K. Dupret, J. Zakrzewski, and D. Delande, Europhys. Lett. 31, 251 (1995)

[5] G. Alber and P. Zoller, Phys. Rep. 199, 231 (1991).

[6] D. Delande, in Chaos and Quantum Physics, edited by M.-J. Giannoni, A. Voros, and J. Zinn-Justin, Les Houches Summer School Session LII (North-Holland, Amsterdam, 1991).
[7] R. V. Jensen, S. M. Susskind, and M. M. Sanders, Phys. Rep. 201, 1 (1991).

[8] H. Friedrich and D. Wintgen, Phys. Rep. 183, 37 (1989).

[9] B. G. Klappauf, W. H. Oskay, D. A. Steck, and M. G. Raizen, Phys. Rev. Lett. 81, 4044 (1998); F. L. Moore, J. C. Robinson, C. Bharucha, P. E. Williams, and M. G. Raizen, ibid. 73, 2974 (1994).

[10] A. M. Herkommer and W. P. Schleich, Comments At. Mol. Phys. 33, 145 (1997).

[11] J. E. Bayfield, Chaos 1, 110 (1991).

[12] T. M. Fromhold, L. Eaves, F. W. Sheard, M. L. Leadbeater, T. J. Foster, and P. C. Main, Phys. Rev. Lett. 72, 2608 (1994).

[13] G. Muller, G. S. Boebinger, H. Mathur, L. N. Pfeiffer, and K. W. West, Phys. Rev. Lett. 75, 2875 (1995). 
[14] M. Domke, K. Schulz, G. Remmers, A. Gutierrez, G. Kaindl, and D. Wintgen, Phys. Rev. A 51, R4309 (1995).

[15] K. Karremans, W. Vassen, and W. Hogervorst, Phys. Rev. Lett. 81, 4843 (1998).

[16] W. Schweizer and W. Jans, Phys. Rev. A 57, 1186 (1998); W. Schweizer, W. Jans, and T. Uzer, ibid. 58, 1382 (1998); M. W. Beims and G. Alber, J. Phys. B 29, 4139 (1996).

[17] A. J. Lichtenberg and M. A. Lieberman, Regular and Stochastic Motion (Springer-Verlag, New York, 1983).

[18] M. Lakshmanan and R. Sahadevan, Phys. Rep. 224, 1 (1993); W. H. Steeb and N. Euler, Nonlinear Evolution Equations and Painlevé Test (World Scientific, Singapore, 1988); M. J. Ablowitz, A. Ramani, and H. Segur, J. Math. Phys. 21, 715 (1980).

[19] F. Cabral and J. A. C. Gallas, Phys. Rev. Lett. 58, 2611 (1987).

[20] Y. Alhassid, E. A. Hinds, and D. Meschede, Phys. Rev. Lett. 59, 1545 (1987).

[21] K. Ganesan and M. Lakshmanan, Phys. Rev. A 42, 3940 (1990).

[22] K. Ganesan and M. Lakshmanan, Phys. Rev. A 48, 964 (1993).

[23] W. Paul, Rev. Mod. Phys. 62, 531 (1990); M. El Ghafar, P. Törmä, V. Savichev, E. Mayr, A. Zeiler, and W. Schleich, Phys. Rev. Lett. 78, 4181 (1997); M. Moore and R. Blümel, Phys. Rev. A 48, 3082 (1993); R. Blümel, J. M. Chen, F. Diedrich, E. Peik, W. Quint, W. Schleich, Y. R. Shen, and H. Walther, Nature (London) 334, 309 (1988).

[24] J. Main and G. Wunner, Phys. Rev. A 55, 1743 (1997); G. Tanner, K. T. Hansen, and J. Main, Nonlinearity 9, 1641 (1996); M. Courtney and D. Kleppner, Phys. Rev. A 53, 178 (1996); M. W. Beims and G. Alber, ibid. 48, 3123 (1993); J. M. Mao and J. B. Delos, ibid. 45, 1746 (1992); H. Friedrich and D. Wintgen, Phys. Rep. 183, 37 (1989).

[25] A. Ramani, B. Dorizzi, and B. Grammaticos, Phys. Rev. Lett. 49, 1539 (1982).

[26] P. J. Redmond, Phys. Rev. 133, 1352 (1964).

[27] O. Halpern, Z. Phys. 18, 287 (1923); O. Klein, ibid. 22, 109 (1924); W. Lenz, ibid. 24, 197 (1924); N. R. Sen, ibid. 56, 673 (1929).
[28] W. Pauli, Z. Phys. 36, 336 (1926), translated in Sources of Quantum Mechanics, edited by B. Van der Waerden (Dover, New York, 1968); discussions of this paper were given by C.E. Wulfman, in Group Theory and its Application II, edited by E. M. Loeb (Academic Press, New York, 1971) and B. G. Adams, Algebraic Approach to Simple Quantum Systems (Springer, Berlin, 1994).

[29] D. M. Fradkin, Prog. Theor. Phys. 37, 789 (1967); L. H. Buch and H. H. Denman, Am. J. Phys. 43, 1046 (1975); W. H. Heintz, ibid. 42, 1078 (1974).

[30] J. B. Delos, S. K. Knudson, and D. W. Noid, Phys. Rev. Lett. 50, 579 (1983); Phys. Rev. A 28, 7 (1983); R. L. Waterland, J. B. Delos, and M. L. Du, ibid. 35, 5064 (1987).

[31] R. Blümel, C. Kappler, W. Quint, and H. Walther, Phys. Rev. A 40, 808 (1989).

[32] G. Baumann and T. F. Nonnenmacher, Phys. Rev. A 46, 2682 (1992).

[33] J. Hietarinta, Phys. Rep. 147, 85 (1987).

[34] J. von Milczewski, D. Farrelly, and T. Uzer, Phys. Rev. Lett. 78, 2349 (1997); R. C. Hilborn, Am. J. Phys. 63, 330 (1995); P. A. Dando, T. S. Monteiro, W. Jans, and W. Schweizer, Prog. Theor. Phys. Suppl. 116, 403 (1994); M. J. Gourlay, T. Uzer, and D. Farrelly, Phys. Rev. A 47, 3113 (1993); J. A. Milligan and D. Farrelly, ibid. 47, 3137 (1993); T. P. Grozdanov and M. J. Raković, J. Phys. B 23, 3531 (1990); H. Friedrich and D. Wintgen, Phys. Rep. 183, 37 (1989).

[35] V. Kondratovich and J. B. Delos, Phys. Rev. A 57, 4654 (1998); 56, R5 (1997); F. Robicheaux and J. Shaw, ibid. 58, 1043 (1998).

[36] M. W. Beims, V. Kondratovich, and J. B. Delos, Phys. Rev. Lett. 81, 4537 (1998).

[37] B. V. Derjaguin, Sci. Am. 203, 47 (1960); C. CohenTannoudji, B. Diu, and F. Laloë, Quantum Mechanics, (Wiley, New York, 1977), Vol. II.

[38] J. Main, M. Schwacke, and G. Wunner, Phys. Rev. A 57, 1149 (1998).

[39] G. Baumann, Phys. Lett. A 162, 464 (1992). 